\title{
Large Shape Staggering in Neutron-Deficient Bi Isotopes
}

A. Barzakhø, ${ }^{1, *}$ A. N. Andreyev, ${ }^{2,3}$ C. Raison, ${ }^{2}$ J. G. Cubiss, ${ }^{2}$ P. Van Duppen, ${ }^{4}$ S. Péru, ${ }^{5}$ S. Hilaire, ${ }^{5}$ S. Goriely, ${ }^{6}$ B. Andel,${ }^{7}$ S. Antalic, ${ }^{7}$ M. Al Monthery, ${ }^{2}$ J. C. Berengut, ${ }^{8}$ J. Bieron,,${ }^{9}$ M. L. Bissell, ${ }^{10}$ A. Borschevsky, ${ }^{11}$ K. Chrysalidis, ${ }^{12,13}$ T. E. Cocolios, ${ }^{4}$ T. Day Goodacre, ${ }^{14,12,10}$ J.-P. Dognon, ${ }^{15}$ M. Elantkowska, ${ }^{16}$ E. Eliav, ${ }^{17}$ G. J. Farooq-Smith,,${ }^{4,}$ D. V. Fedorov, ${ }^{1}$ V. N. Fedosseev, ${ }^{12}$ L. P. Gaffney, ${ }^{18, \$}$ R. F. Garcia Ruiz, ${ }^{10,}$ M. Godefroid, ${ }^{19}$ C. Granados, ${ }^{12,4}$ R. D. Harding ${ }^{2,12}$ R. Heinke, ${ }^{13, \|}$ M. Huyse, ${ }^{4}$ J. Karls, ${ }^{12,20}$ P. Larmonier, ${ }^{12}$ J. G. Li (李冀光) ${ }^{21}$ K. M. Lynch, ${ }^{12}$ D. E. Maison, ${ }^{1,22}$ B. A. Marsh, ${ }^{12}$ P. Molkanov, ${ }^{1}$ P. Mosat, ${ }^{7}$ A. V. Oleynichenko, ${ }^{1,23}$ V. Panteleev, ${ }^{1}$ P. Pyykkö, ${ }^{24}$ M. L. Reitsma, ${ }^{11}$ K. Rezynkina, ${ }^{4}$ R. E. Rossel, ${ }^{12}$ S. Rothe, ${ }^{12}$ J. Ruczkowski, ${ }^{16}$ S. Schiffmann, ${ }^{19}$ C. Seiffert, ${ }^{12}$ M. D. Seliverstov, ${ }^{1}$ S. Sels, ${ }^{4, \|}$ L. V. Skripnikov, ${ }^{1,22}$ M. Stryjczyk,${ }^{4,25}$ D. Studer, ${ }^{13}$ M. Verlinde, ${ }^{4}$ S. Wilman, ${ }^{16}$ and A. V. Zaitsevskii ${ }^{1,23}$

${ }^{1}$ Petersburg Nuclear Physics Institute, NRC Kurchatov Institute, 188300 Gatchina, Russia

${ }^{2}$ Department of Physics, University of York, York YO10 5DD, United Kingdom

${ }^{3}$ Advanced Science Research Center, Japan Atomic Energy Agency, Tokai-mura, Ibaraki 319-1195, Japan

${ }^{4}$ KU Leuven, Instituut voor Kern-en Stralingsfysica, B-3001 Leuven, Belgium

${ }^{5}$ Université Paris-Saclay, CEA, LMCE, 91680 Bruyères-le-Châtel, France

${ }^{6}$ Institut d'Astronomie et d'Astrophysique, CP-226, Université Libre de Bruxelles, 1050 Brussels, Belgium

${ }^{7}$ Department of Nuclear Physics and Biophysics, Comenius University in Bratislava, 84248 Bratislava, Slovakia

${ }^{8}$ School of Physics, University of New South Wales, Sydney NSW 2052, Australia

${ }^{9}$ Instytut Fizyki Teoretycznej, Uniwersytet Jagielloński, ul. prof. Stanistawa Łojasiewicza 11, Kraków, Poland

${ }^{10}$ School of Physics and Astronomy, The University of Manchester, Manchester M13 9PL, United Kingdom

${ }^{11}$ Van Swinderen Institute, University of Groningen, 9747 Groningen, Netherlands

${ }^{12}$ CERN, Esplanade des Particules 1, 1211 Geneva 23, Switzerland

${ }^{13}$ Institut für Physik, Johannes Gutenberg-Universität, Mainz, D-55128 Mainz, Germany

${ }^{14}$ TRIUMF, 4004 Wesbrook Mall, Vancouver British Columbia V6T 2A3, Canada

${ }^{15}$ NIMBE, CEA, CNRS, Universiteé Paris-Saclay, CEA Saclay, 91190 Gif-sur-Yvette, France

${ }^{16}$ Poznan University of Technology, Piotrowo 3, Poznan 60-965, Poland

${ }^{17}$ School of Chemistry, Tel Aviv University, 69978 Tel Aviv, Israel

${ }^{18}$ School of Engineering and Computing, University of the West of Scotland, Paisley PA1 2BE, United Kingdom

${ }^{19}$ SQUARES, CP160/09, Université libre de Bruxelles, Av. F.D. Roosevelt 50, 1050 Brussels, Belgium

${ }^{20}$ Department of Physics, University of Gothenburg, SE-412 96 Gothenburg, Sweden

${ }^{21}$ Institute of Applied Physics and Computational Mathematics, Beijing 100088, China

${ }^{22}$ Saint Petersburg State University, 7/9 Universitetskaya nab., St. Petersburg, 199034 Russia

${ }^{23}$ Department of Chemistry, M. V. Lomonosov Moscow State University, Leninskie gory 1/3, Moscow, 119991 Russia

${ }^{24}$ Department of Chemistry, University of Helsinki, P.O. Box 55 (A. I. Virtasen aukio 1), FIN-00014 Helsinki, Finland

${ }^{25}$ University of Jyväskylä, Department of Physics, P.O. Box 35, FI-40014 Jyväskylä, Finland

(Received 19 August 2021; revised 1 October 2021; accepted 8 October 2021; published 2 November 2021)

The changes in the mean-square charge radius (relative to ${ }^{209} \mathrm{Bi}$ ), magnetic dipole, and electric quadrupole moments of ${ }^{187,188,189,191} \mathrm{Bi}$ were measured using the in-source resonance-ionization spectroscopy technique at ISOLDE (CERN). A large staggering in radii was found in ${ }^{187,188,189} \mathrm{Bi}^{g}$, manifested by a sharp radius increase for the ground state of ${ }^{188} \mathrm{Bi}$ relative to the neighboring ${ }^{187,189} \mathrm{Bi}^{g}$. A large isomer shift was also observed for ${ }^{188} \mathrm{Bi}^{m}$. Both effects happen at the same neutron number, $N=105$, where the shape staggering and a similar isomer shift were observed in the mercury isotopes. Experimental results are reproduced by mean-field calculations where the ground or isomeric states were identified by the blocked quasiparticle configuration compatible with the observed spin, parity, and magnetic moment.

DOI: 10.1103/PhysRevLett.127.192501

Published by the American Physical Society under the terms of the Creative Commons Attribution 4.0 International license. Further distribution of this work must maintain attribution to the author(s) and the published article's title, journal citation, and DOI.
The shape and the size of a nucleus are among its most fundamental properties. Usually, isotopic dependence of nuclear radii is smooth [1,2]; however, at certain neutron numbers there are marked irregularities, e.g., abrupt jumps at $N=60$ and 90 [2-4] or distinct discontinuities at the 
neutron shell closures [2,5-8]. One of the most spectacular examples of such irregularities was found via the isotope shift (IS) measurements of ${ }^{177-186} \mathrm{Hg}(Z=80)$ near the neutron midshell at $N=104$ [9-12]. It was shown that the radii of the $I^{\pi}=1 / 2^{-}$ground states $(\mathrm{gs})$ in ${ }^{181,183,185} \mathrm{Hg}$ are substantially larger relative to their even- $A$ neighbors. This phenomenon was characterized as "one of the most remarkable discoveries in nuclear structure physics in the last 50 years" [13].

Fifty years after this discovery, we present in this Letter the second example of such an unusual behavior, now in the lightest bismuth $(Z=83)$ isotopes. By performing laserspectroscopy studies of ${ }^{187,188,189,191} \mathrm{Bi}$, we found a sharp radius increase for ${ }^{188} \mathrm{Bi}^{g}$, relative to the neighboring ${ }^{187,189} \mathrm{Bi}^{g}$. This dramatic change happens at the same neutron number, $N=105$, where the shape staggering started in the isotonic ${ }^{185} \mathrm{Hg}$.

The experiments were performed at the ISOLDE facility (CERN) [14]. The bismuth nuclei were produced in spallation reactions induced by the $1.4-\mathrm{GeV}$ proton beam from the CERN PS Booster impinging on a thick $\mathrm{UC}_{x}$ target $\left(50 \mathrm{~g} \mathrm{~cm}^{-2}\right.$ of $\left.{ }^{238} \mathrm{U}\right)$. The spallation products effused out of the high-temperature target $(T \approx 2500 \mathrm{~K})$ as neutral atoms into the cavity of the Resonance Ionization Laser Ion Source, RILIS [15]. The bismuth atoms were resonantly ionized when the laser beams were wavelength-tuned to the three-step ionization scheme $[16,17]$. The produced photoions were extracted and accelerated by a $30-\mathrm{kV}$ potential and mass separated by ISOLDE's General Purpose Separator.

The hfs and IS measurements were performed for the first-step transition $6 p^{3}{ }^{4} S_{3 / 2}^{o} \rightarrow 6 p^{2}\left({ }^{3} P_{0}\right) 7 s^{2}[0]_{1 / 2}$ $\left(\lambda_{1}=306.9 \mathrm{~nm}\right)$ by scanning the frequency of the narrow band Titanium Sapphire laser [18], whilst the photoion current was monitored by the detection of characteristic $\alpha$ decays using the Windmill decay station [19]. Examples of the hfs spectra are shown in Fig. 1. It is the ultrasensitive insource resonance-ionization spectroscopy that enables us to study nuclei with the yield less than 1 ion per second [see Fig. 1(d)]. From Fig. 1 one can see that the hfs centroid of ${ }^{188} \mathrm{Bi}^{g}$ is strongly shifted in comparison with its neighbors and its own high-spin isomer. This behavior already demonstrates the essential structural changes in this nucleus (see below).

The positions of the hyperfine components in the hfs spectra are determined by the standard relation [20] with five parameters: nuclear spin $(I)$, isotope shift relative to the stable ${ }^{209} \mathrm{Bi}\left(\delta \nu_{A, 209}\right)$, magnetic hfs constants $\left(a_{1}\right.$ and $\left.a_{2}\right)$ for the first $\left(6 p^{3}{ }^{4} S_{3 / 2}^{o}\right)$ and the second $\left\{6 p^{2}\left({ }^{3} P_{0}\right) 7 s^{2}[0]_{1 / 2}\right\}$ level of the ionization scheme, and electric quadrupole hfs constant $b_{1}$ for the first level. Note that $b_{2} \equiv 0$ since the second level has total electronic angular momentum $J=1 / 2$.

Because of the limited resolution of RILIS, the nuclear spin cannot be determined by just considering number, positions, and relative intensities of individual hyperfine

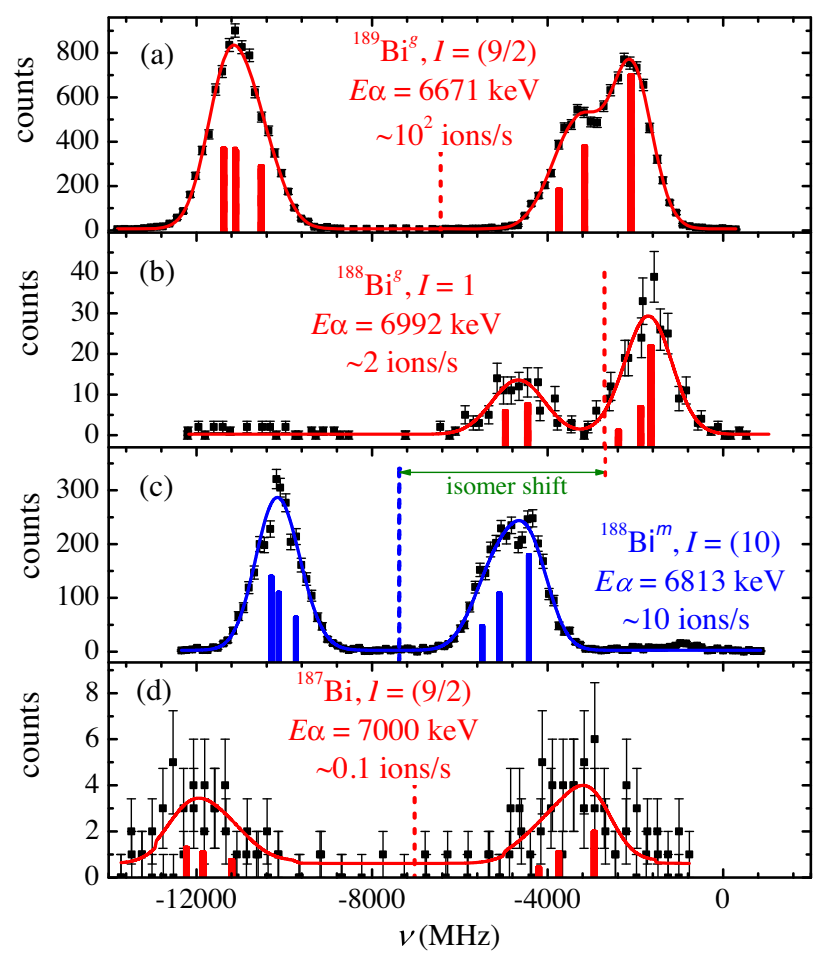

FIG. 1. The hfs spectra of selected Bi isotopes. Nuclear spins, $\alpha$-decay energies, and yields measured at the Windmill decay station are shown for each case. The solid lines depict the Voigtprofile fit to the data. The vertical dashed lines mark the centroids of the corresponding hfs's. The calculated positions and relative intensities of the individual hyperfine components are shown by the vertical bars. The green arrow in panel (c) indicates the isomer shift in ${ }^{188} \mathrm{Bi}$. The zero point on the frequency scale corresponds to a wave number of $32588.16 \mathrm{~cm}^{-1}$.

components. Instead, the "integration method" was used $[17,21]$, which is based on a comparison of the ratio of the areas under each broad peak (see two peaks in each panel of Fig. 1) with theoretically predicted values for different spin assumptions. The theoretical ratio depends only on the nuclear spin: $r_{\text {theor }}=[(I+1) / I] \quad[17,22]$, leading to $r_{\text {theor }}=2,1.5$, and 1.33 for the spin assumption of $I=1,2$, and 3 , respectively. The weighted mean value $r_{\text {expt }}=2.00(12)$ for the six experimental hfs spectra of ${ }^{188} \mathrm{Bi}^{g}$ gives a strong preference for an $I=1$ assignment.

As atomic spectroscopy cannot determine the parity of the nuclear states, we used nuclear-spectroscopic data. Based on the unhindered nature of the 6992-keV $\alpha$ decay of ${ }^{188} \mathrm{Bi}^{g}$ followed by the $117.5-\mathrm{keV} E 1$ decay feeding the adopted (2-) state of the daughter ${ }^{184} \mathrm{Tl}^{\mathrm{m} 2}$ [23], we inferred a positive parity for ${ }^{188} \mathrm{Bi}^{g}$. Thus, the proposed assignment $\left[I^{\pi}=1^{(+)}\right]$should supersede the previous one $\left[I^{\pi}=\left(3^{+}\right)\right]$ made via $\alpha$-decay studies by analogy with heavier even- $A$ Bi nuclei [23]. For ${ }^{187,189} \mathrm{Bi}^{g}$ and ${ }^{188} \mathrm{Bi}^{m}$ the adopted spin values from [24] were used when fitting the hfs spectra $[I=(9 / 2)$ and $I=(10)$, respectively $]$. 
TABLE I. IS $\left(\delta \nu_{A, 209}\right)$, hfs constants $\left(a_{2}, b_{1}\right)$, changes in mean-square charge radius $\left(\delta\left\langle r^{2}\right\rangle_{A, 209}\right)$, and magnetic $(\mu)$ and quadrupole $\left(Q_{s}\right)$ moments for investigated Bi nuclei.

\begin{tabular}{lclllrrr}
\hline \hline$A$ & $I^{\pi}$ & $\delta \nu_{A, 209}(\mathrm{MHz})$ & $\delta\left\langle r^{2}\right\rangle_{A, 209}\left(\mathrm{fm}^{2}\right)^{\mathrm{a}}$ & $a_{2}(\mathrm{MHz})$ & $\mu\left(\mu_{N}\right)^{\mathrm{a}}$ & \multicolumn{1}{c}{$b_{1}(\mathrm{MHz})$} & $Q_{s}(b)$ \\
\hline 187 & $\left(9 / 2^{-}\right)$ & $-22990(250)$ & $-0.949(11)\{47\}$ & $4379(32)$ & $3.638(27)\{20\}$ & $-910(400)$ & $-1.26(55)$ \\
$188 g$ & $1^{(+)}$ & $-8370(160)$ & $-0.335(6)\{17\}$ & $5380(280)$ & $0.994(51)\{20\}$ & $620(270)$ & $+0.85(37)$ \\
$188 m$ & $\left(10^{-}\right)$ & $-23670(100)$ & $-0.978(4)\{49\}$ & $1347(8)$ & $2.464(15)\{10\}$ & $-1220(320)$ & $-1.68(45)$ \\
189 & $\left(9 / 2^{-}\right)$ & $-20823(50)$ & $-0.859(2)\{43\}$ & $4413(7)$ & $3.667(6)\{20\}$ & $-1160(160)$ & $-1.60(23)$ \\
& & $-19900(1100)^{\mathrm{b}}$ & & $4500(330)^{\mathrm{b}}$ & & - \\
191 & $\left(9 / 2^{-}\right)$ & $-19610(50)$ & $-0.810(2)\{40\}$ & $4482(11)$ & $3.724(9)\{20\}$ & $-1023(85)$ & $-1.41(13)$ \\
& & $-19370(230)^{\mathrm{b}}$ & & $4440(60)^{\mathrm{b}}$ & & & - \\
\hline \hline
\end{tabular}

${ }^{a}$ The statistical and systematical uncertainties are shown in round and curly brackets, respectively. The latter values originate from the uncertainty of the $F$ and $M$ factors for $\delta\left\langle r^{2}\right\rangle_{A, 209}$ and from the uncertainty of the hyperfine anomaly for $\mu$ that was estimated using data for heavier bismuth isotopes with the same spin and parity [25,26]. For $\mu\left({ }^{188} \mathrm{Bi}^{g}\right)$ a conservative $2 \%$ uncertainty was additionally included to account for unknown anomaly.

${ }^{\mathrm{b}}$ Reference [27].

Experimental data were fitted by a Voigt profile (more details are in Ref. [17]). In the fit, ratios $\rho=a_{2} / a_{1}$ were fixed according to high-resolution hfs measurements for heavier bismuth isotopes with the same spins as for the aforementioned nuclei [25].

As there are no heavier isotopes with the spin $I=1$, $\rho\left({ }^{188} \mathrm{Bi}^{g}\right)=-11.01$ was fixed (as in ${ }^{209} \mathrm{Bi}$ ), with a possible variation of this value in the limits of $1 \%$ [25]. The deduced $a_{2}$ and $b_{1}$ hfs constants and $\delta \nu_{A, 209}$ are presented in Table I.

Magnetic moments were derived from the hfs constants $a_{2}$ by the standard relation [see Eq. (6) in [2] ]. The following reference values were used: $a_{2,209}=4922.3(20) \mathrm{MHz}$ and $\mu_{209}=4.0900(15) \mu_{N}$ [26]. The magnetic moments are presented in Table I.

The hyperfine constant $b$ is related to the spectroscopic quadrupole moment $Q_{s}$ via equation $b=e Q_{s} \times V$, where $V$ is the electric field gradient (EFG) produced by the electrons at the site of the nucleus. Thus, when $Q_{s}$ is known for a reference isotope ${ }^{209} \mathrm{Bi}$, its value for other isotopes can be determined by the scaling relation

$$
\frac{Q_{s}\left({ }^{A} \mathrm{Bi}\right)}{Q_{s}\left({ }^{209} \mathrm{Bi}\right)}=\frac{b\left({ }^{A} \mathrm{Bi}\right)}{b\left({ }^{209} \mathrm{Bi}\right)} .
$$

There are two independent $Q_{s}$ measurements for ${ }^{209} \mathrm{Bi}$ made in the 1970s: $Q_{s}=-0.37(3) b$ (muonic x ray, Ref. [28]) and $Q_{s}=-0.50(8) b$ (pionic x ray, Ref. [29]). The noticeable difference between these values, their large uncertainties, and a multitude of corrections needed in order to reliably estimate $Q_{s}$ by these methods (Refs. $[28,30]$ ) prevent us from using a muonic or pionic result as a reference value. The alternative way is to calculate the EFG and based on the measured $b\left({ }^{209} \mathrm{Bi}\right)$, deduce $Q_{s}\left({ }^{209} \mathrm{Bi}\right)$. However, the complicated electronic structure makes accurate calculations for atomic bismuth a challenging problem. The first $a b$ initio calculations by the multiconfiguration Dirac-Fock method [31] gave the reference value $Q_{s}\left({ }^{209} \mathrm{Bi}\right)=-0.516(15) b$ adopted in the recent compilation [32]. However, subsequent advanced molecular calculations resulted in $-0.420(8) b$ [33] and $-0.415 b$ [34], questioning this value.

To overcome this longstanding discrepancy, we summarized the results of 33 atomic and molecular calculations for ${ }^{209} \mathrm{Bi}$, either published in the last decade (2013-2021) [33-37], or made specifically for this study. In these calculations a variety of advanced theoretical methods [38-44] with various computational strategies [45-49] were used by several independent groups on five continents. Combining measured values of the hfs constant $b$ of several electronic states in the neutral ${ }^{209} \mathrm{Bi}$ atom, the ${ }^{209} \mathrm{Bi}^{+}$ion, as well as in several diatomic molecules (BiN, BiP, BiF, BiI, $\mathrm{BiO}, \mathrm{BiCl}$ ), with calculated $\mathrm{EFG}$ values for those particular systems, we obtained a sample of 33 values of $Q_{s}\left({ }^{209} \mathrm{Bi}\right)$. The "world average" $Q_{s}\left({ }^{209} \mathrm{Bi}\right)=-0.420(17) b$ was deduced by taking the average of all these results, and the uncertainty was evaluated as a standard deviation of this sample. The quadrupole moments for ${ }^{187,188,189,191} \mathrm{Bi}$ calculated using Eq. (1), "world average" $Q_{s}\left({ }^{209} \mathrm{Bi}\right)$, and $b_{1}\left({ }^{209} \mathrm{Bi}\right)$ from Ref. [50] are presented in Table I. The details of methods and calculations will be published elsewhere.

The electronic factors $F$ and $M$ needed to extract $\delta\left\langle r^{2}\right\rangle$ from the measured IS [see Eqs. $(8,9)$ in Ref. [2]] were determined similarly to the calculations performed in Refs. [51,52]: $F=$ $23.8(10) \mathrm{GHz} \mathrm{fm}^{-2}$ and $M=-750(100) \mathrm{GHz}$ u.

In Fig. 2 the $\delta\left\langle r^{2}\right\rangle_{A, 209}$ values for bismuth nuclei are compared with the data for isotonic lead and mercury isotopes. For all three isotopic chains, one observes the similar smooth trend until $N=106$ along with the small and well-known odd-even staggering (OES). Surprisingly, a huge staggering in radii appears for ${ }^{187-189} \mathrm{Bi}$ isotopes manifested by a drastic increase for ${ }^{188} \mathrm{Bi}^{g}$ in comparison with the neighboring ${ }^{187,189} \mathrm{Bi}^{g}$ and with ${ }^{188} \mathrm{Bi}^{m}$. This dramatic change happens at the same neutron number, $N=105$, where the famous $\mathrm{Hg}$ shape staggering starts $[10,11]$. The latter was interpreted as a sharp change between nearly spherical shapes in the even- $N$ cases and 


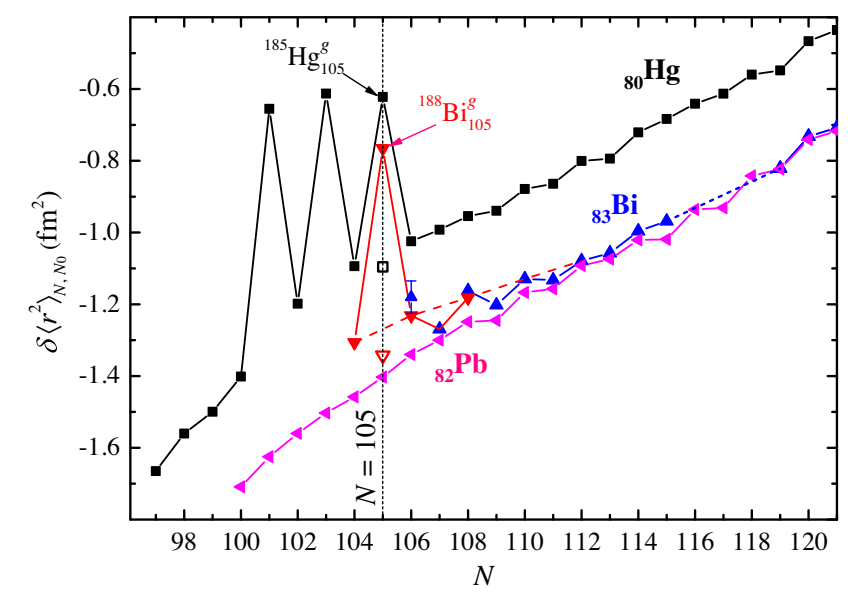

FIG. 2. Changes in the mean-square charge radii for $\mathrm{Bi}$ (downward triangles, present work; upward triangles, Refs. [27,55,56]), Pb (leftward triangles, Refs. [5,57,58]), and $\mathrm{Hg}$ (squares, Refs. [10-12]) isotopes. Full and hollow symboles label the gs's and isomers, respectively. Data for each chain are shifted along the $Y$ axis to improve visibility. Red dashed line connects the data for the even- $N 9 / 2^{-}$Bi gs's to demonstrate deviation from the $\mathrm{Pb}$ trend.

strongly prolate deformed configurations in the odd- $N$ isotopes [53]. In contrast to the mercury isotopes with spin 0 or $1 / 2$ for which $Q_{s} \equiv 0$, for ${ }^{188} \mathrm{Bi}^{g}$ one can directly check this interpretation using the measured $Q_{s}$ value. Indeed, in the strong coupling scheme the quadrupole moment can be related to the quadrupole deformation parameter $\beta$ [see, for example, Eq. (14) in Ref. [54]]. Using this relation one obtains $\beta\left({ }^{188} \mathrm{Bi}^{g}\right)=+0.25(7)$, whereas $\beta\left({ }^{188} \mathrm{Bi}^{m}\right)=-0.08(2), \beta\left({ }^{189} \mathrm{Bi}\right)=-0.10(1)$, and $\beta\left({ }^{187} \mathrm{Bi}\right)=-0.08(4)$. Thus, ${ }^{188} \mathrm{Bi}^{g}$ is strongly prolate deformed, whilst its $10^{-}$isomer and the adjacent isotopes with lower radii have a small deformation.

It was noted in Ref. [27] that the $\delta\left\langle r^{2}\right\rangle$ values of the 9/2$\mathrm{Bi}$ gs's follow the same trend as the radii of the spherical $\mathrm{Pb}$ isotopes down to $N=112$, but start to deviate at $N=110$. This was interpreted as a possible onset of deformation in ${ }^{193,191} \mathrm{Bi}^{g}$. Our new data confirm this deviation from sphericity down to ${ }^{187} \mathrm{Bi}(N=104)$. This is consistent with suggestions from extensive nuclear-spectroscopy data on the possible structural changes in the $N \leq 110$ bismuth isotopes (see Refs. [23,59-63]).

The experimental results were analyzed on the basis of Hartree-Fock-Bogoliubov (HFB) calculations with the D1M Gogny interaction and the equal filling approximation for the odd- $A$ and odd-odd nuclei. Potential energy surfaces (PESs) were calculated with the blocked quasiparticles (qp) compatible with the experimental spin and parity. The total number of PESs to compute is therefore particularly large for odd-odd systems, with, e.g., 100 PESs in ${ }^{188} \mathrm{Bi}$ with minima located at most $1 \mathrm{MeV}$ above the HFB gs.

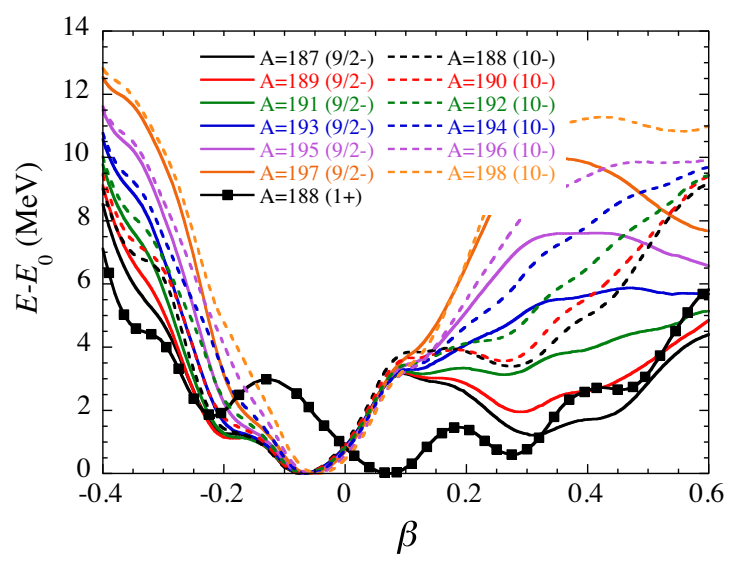

FIG. 3. HFB PESs obtained by blocking the first $9 / 2^{-}$qp in odd- $A$ bismuth isotopes (solid lines), $10^{-}$in even- $A$ ones (dashed lines), and $1^{+}$in ${ }^{188} \mathrm{Bi}$ (squares). $E_{0}$ is the minimal energy of the corresponding PES. For each PES, at least one of the minima has a magnetic moment compatible with experimental data. For $9 / 2^{-}$ and $10^{-}$states it is a minimum at $\beta \approx-0.07$, whereas for $1^{+}$state in ${ }^{188} \mathrm{Bi}$ it is a minimum at $\beta \approx+0.28$.

The further selection of the proper states was made by constraining the calculated magnetic moment to the experimental value. The magnetic moments were calculated as detailed in Ref. [64] on the basis of an effective magnetic moment operator $\hat{\mu}^{\text {eff }}=0.82 \times g_{s} \hat{s}+1.25 \times g_{l} \hat{l}$. The beyond mean-field and core-polarization effects were taken into account via the adopted effective coefficients (see Ref. [65]) in order to best reproduce all experimental moments [66]. For strongly deformed nuclei, a rotational contribution to the magnetic moment was also taken into account [64]. Following this approach, for a given combination (isotope, configuration) only the level with the lowest energy and a magnetic moment compatible with the experimental value within 50\% was kept. The PESs obtained with this blocking and selection technique are shown in Fig. 3.

For the $9 / 2^{-}$and $10^{-}$states at $A \geq 193$, only a single nearspherical minimum exists. In the lighter isotopes additional oblate and prolate minima appear, but they are still quite flat and shallow. In contrast, the $1^{+}$state in ${ }^{188} \mathrm{Bi}$ corresponds to the well-pronounced prolate minimum at $\beta=0.28$ [compare with $\beta=0.25(7)$ extracted from the $Q_{s}$ value]. The structure of this $1^{+}$state is determined by the blocked configuration $\pi 1 / 2[530] \otimes \nu 1 / 2[521]$, which is the oddodd counterpart of the $\nu 1 / 2[521]$ configuration in the isotonic ${ }^{185} \mathrm{Hg}^{g}$.

Note that the $1^{+}$minimum at $\beta_{2} \simeq 0.08$, despite being lower than the $\beta=0.28$ minimum, has a magnetic moment of $\mu^{\text {eff }}=0.0 \mu_{N}$ incompatible with experimental data and can therefore not be retained as a potential candidate for the gs configuration.

The calculated magnetic moments and $\delta\left\langle r^{2}\right\rangle$ values obtained from the HFB minima constrained by the 


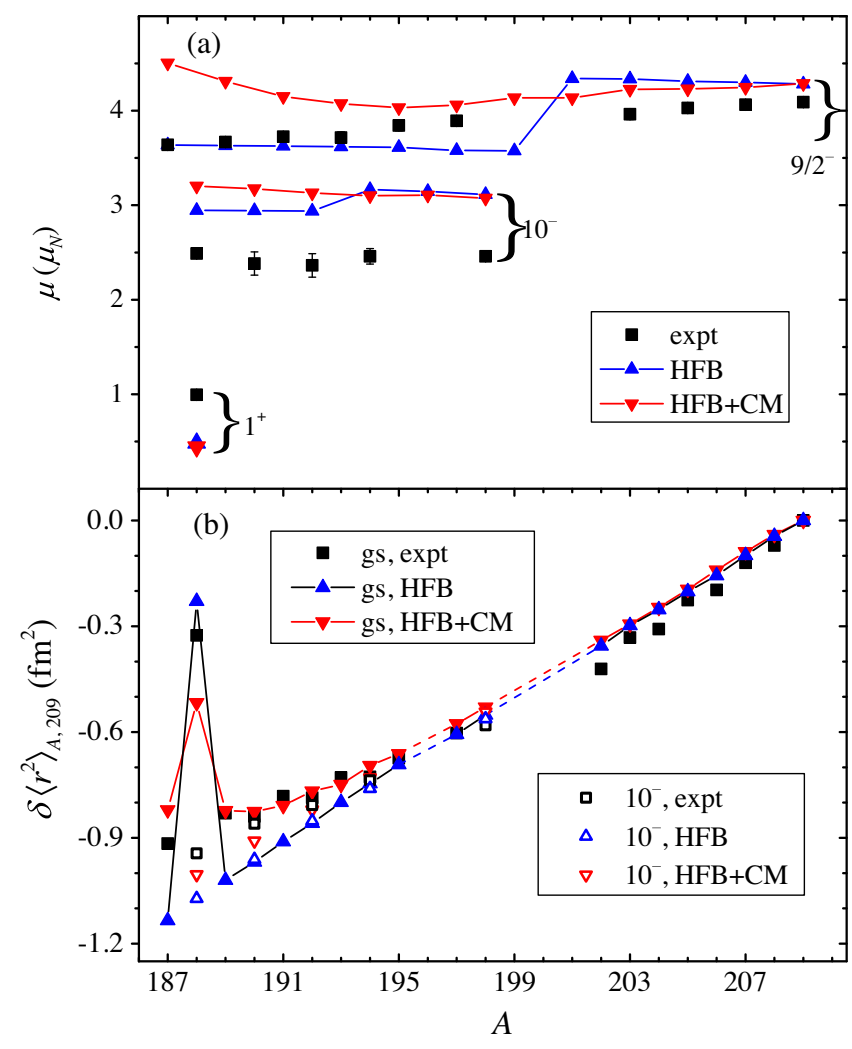

FIG. 4. (a) Comparison between experimental and theoretical magnetic moments for the various bismuth nuclei. Black squares correspond to experimental data, blue upward triangles to the HFB minima, and red downward triangles to the CM calculations. The magnetic moments are shown for the $9 / 2^{-}, 10^{-}$states and the $1^{+}$gs in ${ }^{188} \mathrm{Bi}$. (b) Same for the gs $\delta\left\langle r^{2}\right\rangle_{A, 209}$. The open symbols correspond to the $10^{-}$isomers.

magnetic moment (at $\beta \approx-0.07$ for $9 / 2^{-}$and $10^{-}$states and at $\beta \approx+0.28$ for $1^{+}$state) are compared with experimental data in Fig. 4. As shown in Fig. 4(b), the large $\delta\left\langle r^{2}\right\rangle$ $\left({ }^{188} \mathrm{Bi}^{g}\right)$ value is well reproduced; however, the theory overestimates the slope of the $\delta\left\langle r^{2}\right\rangle$ isotopic dependency for the $9 / 2^{-}$and $10^{-}$states at $A \leq 193$. Such a discrepancy suggests possible configuration mixing (CM) between the various low-lying states connected with different minima in PES. Indeed, it is this kind of CM that was invoked to explain a small deviation from the spherical droplet model predictions in the core $\mathrm{Pb}$ nuclei around $N=104$ [57]. However, such a CM for odd- $A$ or odd-odd nuclei cannot currently be modeled microscopically. Therefore, in order to mimic such a mixing between prolate, oblate, and near spherical shapes, a phenomenological approach, inspired from statistical physics $[67,68]$, was followed. It consists of calculating the average value of the observable $\mathcal{O}$ by mixing its values over the various states $q$ with different deformations, through the expression

$$
\langle\mathcal{O}\rangle=\frac{\int_{q} \mathcal{O} \exp (-E / T) d q}{\int_{q} \exp (-E / T) d q},
$$

where $E$ is the HFB energy along a given PES (Fig. 3) and $T$ is a free parameter which enables the mixing of various low-lying configurations. A constant value of $T=1 \mathrm{MeV}$ was chosen in order to improve the description of the charge radii in neutron-deficient bismuth isotopes.

As seen in Fig. 4(b) the mixing procedure preserves the large staggering in the neighborhood of ${ }^{188} \mathrm{Bi}$ but reduces its amplitude. The CM effect may consequently be overestimated for the $1^{+}$state. Only future beyond-mean-field calculations will be able to shed light on the exact impact of $\mathrm{CM}$ and in particular how it affects different blocked configurations. Note that $\mathrm{CM}$ does not significantly influence the $\mu$ values [see Fig. 4(a)]. At the same time, it results in a better agreement of the calculated $\delta\left\langle r^{2}\right\rangle$ of the lightest bismuth isotopes with the experimental data. In particular, it reproduces the observed OES between the $9 / 2^{-}$and $10^{-}$ states for light bismuth isotopes which is absent in the nonmixed HFB approach [see Fig. 4(b)]. Indeed, the nonmixed HFB minima predict the bismuth gs (apart from ${ }^{188} \mathrm{Bi}^{g}$ ) to be quasispherical (Fig. 3), while the CM takes the contributions of the prolate and oblate minima into account, leading to a deviation from sphericity, hence an increase in $\delta\left\langle r^{2}\right\rangle$. The heaviest bismuth isotopes are not as affected by mixing since the PES becomes stiffer when approaching the $N=126$ closed shell (Fig. 3). The reproduction of the OES for the $9 / 2^{-}$and $10^{-}$states can be understood from contributions of the prolate wells that alter between these states for the light bismuth isotopes. The larger stiffness of the PES of the $10^{-}$states compared to the $9 / 2^{-}$states leads to less mixing for the former.

To summarize, ISs and hfs's were studied for neutrondeficient bismuth isotopes using the ultrasensitive (down to $0.1 \mathrm{ion} / \mathrm{s}$ ) in-source resonance-ionization spectroscopy technique. A striking staggering in radii was observed for ${ }^{188} \mathrm{Bi}^{g}$ relative to ${ }^{187,189} \mathrm{Bi}^{g}$ at the same neutron number $(N=105)$ as in the $\mathrm{Hg}$ case (see Refs. [10,11]). It is only the second example of such unusual behavior throughout the nuclide chart. The quadrupole moment of ${ }^{188} \mathrm{Bi}^{g}$ confirms the strong prolate deformation in this nucleus with the newly established spin and parity of $I^{\pi}=1^{(+)}$.

This staggering was successfully explained by HFB calculations, where the ground state is identified by the blocked quasiparticle configuration compatible with the observed spin, parity, and magnetic moment. The departure from the trend for radii of $\mathrm{Pb}$ isotopes, found in light Bi's, was explained by invoking $\mathrm{CM}$ with states of different deformations.

We thank the ISOLDE Collaboration for providing excellent beams and the GSI Target Group for manufacturing the carbon foils for the Windmill. A. B. and M. L. R. would like to thank the Center for Information Technology of the University of Groningen for providing access to the Peregrine high performance computing cluster and for their technical support. S. G. acknowledges financial support from FNRS (Belgium). J.G. L. acknowledges financial 
support by the National Natural Science Foundation of China under Grant No. 11874090. M. St. acknowledges the funding from the European Union's Horizon 2020 research and innovation program under Grant Agreement No. 771036 (ERC CoG MAIDEN). This work was supported by the Fonds de la Recherche Scientifique (F. R. S.-FNRS) and the Fonds Wetenschappelijk Onderzoek-Vlaanderen (FWO) under the EOS Project No. O022818F, by GOA/2015/010 (BOF KU Leuven), the Interuniversity Attraction Poles Programme initiated by the Belgian Science Policy Office (BriX network P7/12), by the ENSAR2: European Union's Horizon 2020 research and innovation program under Grant Agreement No. 654002, by the U.K. Science and Technology Facilities Council, by the Slovak Research and Development Agency (Contract No. APVV-18-0268), by the Slovak grant agency VEGA (Contract No. 1/0651/21), by RFBR according to the research project N 19-02-00005, by the Russian Science Foundation (Grant No. 19-72-10019), by RFBR according to the research project N 20-32-70177, by the foundation for the advancement of theoretical physics and mathematics "BASIS" grant according to Projects No. 21-1-2-47-1 and No. 20-1-5-76-1.

*barzakh_ae@pnpi.nrcki.ru

${ }^{\dagger}$ Present address: Department of Oncology Physics, Edinburgh Cancer Centre, Western General Hospital, Crewe Road South, Edinburgh EH4 2XU, United Kingdom.

${ }^{*}$ Present address: Oliver Lodge Laboratory, University of Liverpool, Liverpool L69 7ZE, United Kingdom.

${ }^{\S}$ Present address: Department of Physics, Massachusetts Institute of Technology, Cambridge, USA.

"Present address: CERN, Esplanade des Particules 1, 1211 Geneva 23, Switzerland.

[1] I. Angeli and K. Marinova, At. Data Nucl. Data Tables 99, 69 (2013).

[2] P. Campbell, I. Moore, and M. Pearson, Prog. Part. Nucl. Phys. 86, 127 (2016).

[3] C. Thibault, F. Touchard, S. Büttgenbach, R. Klapisch, M. de Saint Simon, H. T. Duong, P. Jacquinot, P. Juncar, S. Liberman, P. Pillet, J. Pinard, J. L. Vialle, A. Pesnelle, and G. Huber, Phys. Rev. C 23, 2720 (1981).

[4] G. D. Alkhazov, A. E. Barzakh, V. P. Denisov, V. S. Ivanov, I. Y. Chubukov, V. S. Letokhov, V. I. Mishin, S. K. Sekatsky, and V. N. Fedoseyev, Z. Phys. A 337, 367 (1990).

[5] M. Anselment, W. Faubel, S. Göring, A. Hanser, G. Meisel, H. Rebel, and G. Schatz, Nucl. Phys. A451, 471 (1986).

[6] C. Gorges et al., Phys. Rev. Lett. 122, 192502 (2019).

[7] T. Day Goodacre et al., Phys. Rev. Lett. 126, 032502 (2021).

[8] M. Reponen et al., Nat. Commun. 12, 4596 (2021).

[9] J. Bonn, G. Huber, H.-J. Kluge, L. Kugler, and E. Otten, Phys. Lett. 38B, 308 (1972).

[10] G. Ulm, S. K. Bhattacherjee, P. Dabkiewicz, G. Huber, H. Kluge, T. Kühl, H. Lochmann, E. Otten, and K. Wendt, Z. Phys. A 325, 247 (1986).

[11] B. A. Marsh et al., Nat. Phys. 14, 1163 (2018).

[12] S. Sels et al., Phys. Rev. C 99, 044306 (2019).
[13] K. Heyde and J. L. Wood, Phys. Scr. 91, 083008 (2016).

[14] R. Catherall, W. Andreazza, M. Breitenfeldt, A. Dorsival, G. J. Focker, T. P. Gharsa, G. T. J, J.-L. Grenard, F. Locci, P. Martins, S. Marzari, J. Schipper, A. Shornikov, and T. Stora, J. Phys. G 44, 094002 (2017).

[15] V. Fedosseev, K. Chrysalidis, T. D. Goodacre, B. Marsh, S. Rothe, C. Seiffert, and K. Wendt, J. Phys. G 44, 084006 (2017).

[16] S. Rothe, T. Day Goodacre, D. Fedorov, V. Fedosseev, B. Marsh, P. Molkanov, R. Rossel, M. Seliverstov, M. Veinhard, and K. Wendt, Nucl. Instrum. Methods Phys. Res., Sect. B 376, 91 (2016).

[17] M. Seliverstov, A. Barzakh, R. Ahmed, K. Chrysalidis, T. D. Goodacre, D. Fedorov, V. Fedoseev, C. Granados, B. Marsh, P. Molkanov, V. Panteleev, R. E. Rossel, S. Rothe, S. Wilkins, and IS608 Collaboration, Hyperfine Interact. 241, 40 (2020).

[18] S. Rothe, V. N. Fedosseev, T. Kron, B. A. Marsh, R. E. Rossel, and K. D. A. Wendt, Nucl. Instrum. Methods Phys. Res., Sect. B 317, 561 (2013).

[19] M. D. Seliverstov et al., Phys. Rev. C 89, 034323 (2014).

[20] E. W. Otten, in Treatise on Heavy Ion Science: Volume 8: Nuclear Far From Stability, edited by D. A. Bromley (Springer, Boston, MA, 1989), pp. 517-638.

[21] R. D. Harding et al., Phys. Rev. C 102, 024312 (2020).

[22] G. J. Farooq-Smith, Exploring the magical tenacity of doubly closed-core nuclei with gallium and francium isotopes, Ph.D. thesis, KU Leuven, 2019.

[23] A. N. Andreyev, D. Ackermann, S. Antalic, H. Boardman, P. Cagarda, J. Gerl, F. Heßberger, S. Hofmann, M. Huyse, D. Karlgren et al., Eur. Phys. J. A 18, 39 (2003).

[24] https://www.nndc.bnl.gov/ensdf/, [Online; accessed 04.07.2021].

[25] M. Bissel (unpublished).

[26] S. Schmidt, J. Billowes, M. Bissell, K. Blaum, R. Garcia Ruiz, H. Heylen, S. Malbrunot-Ettenauer, G. Neyens, W. Nörtershäuser, G. Plunien, S. Sailer, V. Shabaev, L. Skripnikov, I. Tupitsyn, A. Volotka, and X. Yang, Phys. Lett. B 779, 324 (2018).

[27] A. E. Barzakh, D. V. Fedorov, V. S. Ivanov, P. L. Molkanov, F. V. Moroz, S. Y. Orlov, V. N. Panteleev, M. D. Seliverstov, and Y. M. Volkov, Phys. Rev. C 95, 044324 (2017).

[28] W. Lee, M. Chen, S. Cheng, E. Macagno, A. Rushton, and C. Wu, Nucl. Phys. A181, 14 (1972).

[29] R. Beetz, F. de Boer, J. K. Panman, J. Konijn, P. Pavlopoulos, G. Tibell, K. Zioutas, I. Bergström, K. Fransson, L. Tauscher, P. Blüm, R. Guigas, H. Koch, H. Poth, and L. M. Simons, Z. Phys. A 286, 215 (1978).

[30] R. Beetz, F. de Boer, K. Fransson, J. Konijn, J. Panman, L. Tauscher, and G. Tibell, Nucl. Phys. A300, 369 (1978).

[31] J. Bieroń and P. Pyykkö, Phys. Rev. Lett. 87, 133003 (2001).

[32] N. Stone, At. Data Nucl. Data Tables 111-112, 1 (2016).

[33] T. Q. Teodoro and R. L. A. Haiduke, Phys. Rev. A 88, 052504 (2013).

[34] A. Shee, L. Visscher, and T. Saue, J. Chem. Phys. 145, 184107 (2016).

[35] J. Liu, Y. Shen, A. Asthana, and L. Cheng, J. Chem. Phys. 148, 034106 (2018).

[36] J. Liu, X. Zheng, A. Asthana, C. Zhang, and L. Cheng, J. Chem. Phys. 154, 064110 (2021). 
[37] L. V. Skripnikov, A. V. Oleynichenko, A. V. Zaitsevskii, D. E. Maison, and A.E. Barzakh, Phys. Rev. C 104, 034316 (2021).

[38] I. P. Grant, Relativistic Quantum Theory of Atoms and Molecules: Theory and Computation (Springer, New York, 2007).

[39] C. Froese Fischer, M. Godefroid, T. Brage, P. Jönsson, and G. Gaigalas, J. Phys. B: At., Mol., Opt. Phys. 49, 182004 (2016).

[40] C. Froese Fischer, G. Gaigalas, P. Jönsson, and J. Bieroń, Comput. Phys. Commun. 237, 184 (2019).

[41] M. Elantkowska, J. Ruczkowski, and J. Dembczyński, Eur. Phys. J. Plus 130, 14 (2015).

[42] M. Kállay, P. G. Szalay, and P. R. Surján, J. Chem. Phys. 117, 980 (2002).

[43] V. A. Dzuba, V. V. Flambaum, P. G. Silvestrov, and O. P. Sushkov, J. Phys. B 18, 597 (1985).

[44] V. A. Dzuba, V. V. Flambaum, P. G. Silvestrov, and O. P. Sushkov, J. Phys. B: At., Mol., Opt. Phys. 20, 1399 (1987).

[45] J. Bieroń, L. Filippin, G. Gaigalas, M. Godefroid, P. Jönsson, and P. Pyykkö, Phys. Rev. A 97, 062505 (2018).

[46] J. Bieroń, C. Froese Fischer, P. Indelicato, P. Jönsson, and P. Pyykkö, Phys. Rev. A 79, 052502 (2009).

[47] A. Papoulia, S. Schiffmann, J. Bieroń, G. Gaigalas, M. Godefroid, Z. Harman, P. Jönsson, N. S. Oreshkina, P. Pyykkö, and I. I. Tupitsyn, Phys. Rev. A 103, 022815 (2021).

[48] M. Elantkowska, J. Ruczkowski, S. Wilman, and M. Suski, Eur. Phys. J. Plus 136, 330 (2021).

[49] L. V. Skripnikov and A. V. Titov, J. Chem. Phys. 142, 024301 (2015).

[50] R. J. Hull and G. O. Brink, Phys. Rev. A 1, 685 (1970).

[51] F. P. Gustafsson et al., Phys. Rev. A 102, 052812 (2020).
[52] E. Kahl and J. Berengut, Comput. Phys. Commun. 238, 232 (2019).

[53] J. Bonn, G. Huber, H.-J. Kluge, and E. Otten, Z. Phys. A 276, 203 (1976).

[54] J. G. Cubiss et al., Phys. Rev. C 97, 054327 (2018).

[55] M. R. Pearson, P. Campbell, K. Leerungnavarat, J. Billowes, I. S. Grant, M. Keim, J. Kilgallon, I. D. Moore, R. Neugart, M. Neuroth, S. Wilbert, and the ISOLDE Collaboration, J. Phys. G 26, 1829 (2000).

[56] A. E. Barzakh, D. V. Fedorov, V. S. Ivanov, P. L. Molkanov, F. V. Moroz, S. Y. Orlov, V. N. Panteleev, M. D. Seliverstov, and Y. M. Volkov, Phys. Rev. C 94, 024334 (2016).

[57] H. De Witte et al., Phys. Rev. Lett. 98, 112502 (2007).

[58] M. D. Seliverstov, A. Andreyev, N. Barré, A. Barzakh, S. Dean, H. De Witte, D. Fedorov, V. Fedoseyev, L. Fraile, S. Franchoo et al., Eur. Phys. J. A 41, 315 (2009).

[59] A. N. Andreyev, D. Ackermann, F. P. Heßberger, K. Heyde, S. Hofmann, M. Huyse, D. Karlgren, I. Kojouharov, B. Kindler, B. Lommel, G. Münzenberg, R. D. Page, K. Van de Vel, P. Van Duppen, W. B. Walters, and R. Wyss, Phys. Rev. C 69, 054308 (2004).

[60] A. Hürstel et al., Eur. Phys. J. A 21, 365 (2004).

[61] A. Herzán̆ et al., Phys. Rev. C 92, 044310 (2015).

[62] H. Pai et al., Phys. Rev. C 85, 064317 (2012).

[63] M. Nyman et al., Eur. Phys. J. A 51, 31 (2015).

[64] S. Péru, S. Hilaire, S. Goriely, and M. Martini, Phys. Rev. C 104, 024328 (2021).

[65] S. Hilaire, S. Péru, and S. Goriely (unpublished).

[66] N. Stone, At. Data Nucl. Data Tables 90, 75 (2005).

[67] V. Martin, J. L. Egido, and L. M. Robledo, Phys. Rev. C 68, 034327 (2003).

[68] S. Hilaire, M. Girod, S. Goriely, and A. J. Koning, Phys. Rev. C 86, 064317 (2012). 